\title{
The Open, the Closed, and the Empty: Time-Resolved Fluorescence Spectroscopy and Computational Analysis of RC-LH1 Complexes from Rhodopseudomonas palustris
}

\author{
Sebastian R. Beyer, ${ }^{\dagger, \|}$ Lars Müller, ${ }^{\ddagger}, \|$ June Southall, ${ }^{\S}$ Richard J. Cogdell, ${ }^{\S}$ G. Matthias Ullmann, ${ }^{* \dagger}$ \\ and Jürgen Köhler* ${ }^{*}$

\begin{abstract}
${ }^{\dagger}$ Experimental Physics IV and Bayreuther Institut für Makromolekülforschung (BIMF), University of Bayreuth, 95440 Bayreuth, Germany

${ }^{\ddagger}$ Computational Biochemistry/Bioinformatics, University of Bayreuth, 95440 Bayreuth, Germany

${ }^{\S}$ Institute of Molecular, Cell and Systems Biology, College of Medical Veterinary and Life Sciences, Biomedical Research Building, University of Glasgow, Glasgow G12 8QQ, Scotland, United Kingdom
\end{abstract}

Supporting Information

ABSTRACT: We studied the time-resolved fluorescence of isolated RC-LH1 complexes from Rhodopseudomonas palustris as a function of the photon fluence and the repetition rate of the excitation laser. Both parameters were varied systematically over 3 orders of magnitude. On the basis of a microstate description we developed a quantitative model for RC-LH1 and obtained very good agreement between experiments and elaborate simulations based on a global master equation approach. The model allows us to predict the relative population of RC-LH1 complexes with the special pair in the neutral state or in the oxidized state $\mathrm{P}^{+}$and those complexes that lack a reaction center.

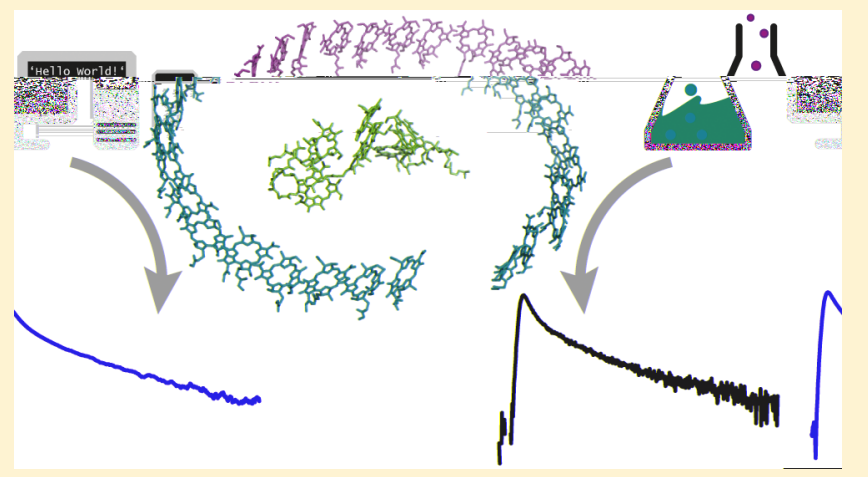

\section{INTRODUCTION}

Photosynthetic purple bacteria have evolved a wonderful modular principle for the light-harvesting ( $\mathrm{LH}$ ) apparatus that captures the solar radiation. These modules consist of pairs of hydrophobic, low molecular weight polypeptides that noncovalently bind a small number of bacteriochlorophyll (Bchl $a$ ) and carotenoid (Car) molecules and that self-assemble to produce the native pigment-protein complexes. ${ }^{1-6}$ Most purple bacteria have two main types of complexes, the core complex, RC-LH1, and the peripheral complex, LH2. In the photosynthetic membrane the $\mathrm{LH} 2$ complexes are arranged in a two-dimensional network around the perimeter of the RC-LH1 complexes and the light energy absorbed by $\mathrm{LH} 2$ is transferred via $\mathrm{LH} 1$ to the reaction center (RC) where it is used to separate charge across the membrane. ${ }^{7}$ For many years, high-resolution structural information has been available for the $\mathrm{RC},^{8-11}$ and the peripheral light-harvesting complexes ${ }^{1-3}$ for a couple of species, whereas the first higher-resolution X-ray structure for a RC-LH1 complex has been determined only recently. ${ }^{6}$ The current view is that there are at least two distinct classes of RCLH1 complexes: Those that are monomeric, i.e., consisting of one RC surrounded by one LH1 complex, and those that are dimeric. ${ }^{12,13}$ For RC-LH1 from Rps. palustris exists a lowresolution X-ray structure. ${ }^{4}$ Accordingly, the $\mathrm{RC}$ is enclosed by an overall elliptically shaped LH1 consisting of $15 \alpha \beta$-subunits that feature a gap, which is also consistent with data from optical single-molecule experiments. ${ }^{14,15}$ Each subunit accommodates two light harvesting $\mathrm{BChl} a$ molecules giving rise to an absorption band at $875 \mathrm{~nm}$. The reaction center complex accommodates a BChl $a$ dimer (special pair, primary donor, $\mathrm{P}$ ), two accessory $\mathrm{BChl} a$ molecules $\left(\mathrm{B}_{\mathrm{A}}, \mathrm{B}_{\mathrm{B}}\right)$, two bacteriopheophytin $a$ (BPheo $a$ ) molecules $\left(\mathrm{H}_{\mathrm{A}}, \mathrm{H}_{\mathrm{B}}\right)$, and two ubiquinones $\left(Q_{A}, Q_{B}\right)$ bound to two protein subunits denoted $L$ and $M$. The cofactors are arranged in two nearly identical branches, called A and $\mathrm{B}$, which share the primary donor, $\mathrm{P} .{ }^{9,11,16,17}$ In particular the RC from Rhodobacter ( $R b$.) sphaeroides has served as a cornerstone for elucidating structure-function relationships employing a large variety of spin resonance ${ }^{18-23}$ and optical spectroscopies. ${ }^{24-31}$ From these studies it is well established that in the RC the absorbed photon-energy is used to drive a series of electron transfer reactions until the secondary ubiquinone $\left(\mathrm{Q}_{B}\right)$ has been fully reduced to $\mathrm{Q}_{B} \mathrm{H}_{2}$; see Figure $1.32,33$

The dynamics of the energy transfer between the LH1 and the RC has been the subject of countless studies, and the characteristic time constants that have been found range from several picoseconds up to a few nanoseconds. ${ }^{34-45}$ In some studies the fluorescent transients were described by up to five

Received: October 28, 2014

Revised: December 17, 2014

Published: December 19, 2014 


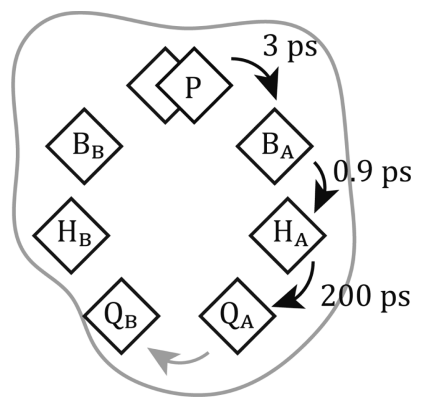

Figure 1. Sketch of the cofactor arrangement in the reaction centers of purple bacteria. The pigments in the two branches $\mathrm{A}$ and $\mathrm{B}$ are represented as diamonds and denote $\mathrm{P}$ (special pair), accessory $\mathrm{BChl}$ a molecules $(\mathrm{B})$, BPheo a $(\mathrm{H})$, and ubiquinone $(\mathrm{Q})$, whereas the protein backbone is indicated by the gray line. The arrows indicate the electron transfer pathway between the cofactors. The time constants for these processes have been taken from ref 32 and are valid for $R b$. sphaeroides.

decay times that could not always be attributed to distinct processes. The general picture that has emerged from these studies can be summarized as follows. Time constants up to 10 ps are associated with the detrapping, i.e., energy transfer from an excited special pair back to the LH1 ring. ${ }^{46-50}$ Energy transfer from the LH1 ring to the neutral special pair takes place with time constants around $30-70 \mathrm{ps},{ }^{48,51-53}$ whereas time constants between 200 and 300 ps were attributed to energy transfer from LH1 to the reduced special pair in the RC. ${ }^{51-55}$ Decay times between 500 ps and 1 ns have been attributed to the decay of excitations on the LH1 ring, ${ }^{56-58}$ and decay times longer than that were sometimes interpreted as a charge recombination in the $\mathrm{RC}$ which results in delayed fluorescence. ${ }^{49,59,60}$ In order to assess these data one has to consider that, due to the lack of isolated, purified RC-LH1 complexes at that time, many of these studies have been carried out on whole chromatophores from purple bacteria. Hence, either some of these samples contained also an unknown fraction of LH2 complexes, or species were used that did not produce peripheral antenna complexes. In any case intercomplex energy transfer between the LH1 and/or LH2 complexes and the influence of concomitant singlet-singlet annihilation on the observed dynamics could not be controlled. To the best of our knowledge, exploiting the benefits of isolation and purification protocols for $\mathrm{RC}-\mathrm{LH} 1$ has led only to one timeresolved pump-probe experiment to date. ${ }^{61}$

Recently, we have investigated systematically the dynamics of isolated LH2 complexes as a function of the excitation conditions. Combining the experimental results with dynamic Monte Carlo simulations we obtained a quantitative understanding of the fluorescence quenching mechanisms in $\mathrm{LH} 2$ without the need for any free parameter. ${ }^{62}$ Moreover, using these results as input for the interpretation of the transients obtained on reconstituted homo-arrays of $\mathrm{LH} 2$ complexes, i.e., model membranes of well-defined composition, we revealed a clear influence of the size and the geometry of the $\mathrm{LH} 2$ clusters on the fluorescence transients. ${ }^{63}$

Here we present a study on the time-resolved emission from isolated RC-LH1 complexes from the species Rps. palustris, where we have systematically varied the photon fluence, the repetition rate of the excitation laser, and the concentrations of a reducing and an oxidizing agent. The data obtained serve as input for a model that allows us to predict quantitatively the time-resolved fluorescence response of isolated RC-LH1 complexes from Rps. palustris over a wide range of the abovementioned parameters.

\section{MATERIALS AND METHODS}

Samples. RC-LH1 complexes from Rps. palustris were isolated and purified as described previously. ${ }^{4}$ After purification, the RC-LH1 complexes were transferred to a buffer containing $20 \mathrm{mM}$ Tris (tris-hydroxymethylaminomethane, Sigma-Aldrich, St. Louis, U.S.A.) at pH 8 and $0.02 \%$ DDM ( $n$-Dodecyl $\beta$-Dmoltoside, Roth, Karlsruhe, Germany). In order to avoid multiple thaw-freeze cycles the material was aliquoted in small amounts and stored at $-80{ }^{\circ} \mathrm{C}$ until used. For the optical experiments this solution was further diluted with buffer to yield an optical density of $0.1 \mathrm{~cm}^{-1}$ at $879 \mathrm{~nm}$. All preparations were made under minimal illumination conditions, and the measurements started immediately after the preparation.

Chemical Manipulation. For reference purposes we conducted experiments where the time-resolved fluorescence response of RC-LH1 complexes was investigated as a function of the concentration of either a reducing or an oxidizing agent. For the reduction of the RC we used 2,3,5,6-tetramethyl- $p$ phenylenediamine (DAD; Sigma-Aldrich, St. Louis, U.S.A.) as reducing agent. A stock solution of $100 \mathrm{mM}$ DAD in ethanol (Sigma-Aldrich, St. Louis, U.S.A.) was added to the RC-LH1 solution until the desired final concentration in the sample was achieved. Oxidation of the RC was accomplished by using potassium hexacyanoferrate(III) (Sigma-Aldrich, St. Louis, U.S.A.) as an oxidant. A stock solution of $100 \mathrm{mM}$ oxidant in $100 \mathrm{mM}$ Tris at $\mathrm{pH} 8$ was added to the RC-LH1 solution until the desired final concentration in the sample was achieved.

Optical Setup. For time-resolved measurements, the samples were illuminated with a pulsed titanium:sapphire laser (Tsunami, Spectra Physics) that was pumped with a frequency doubled $\mathrm{Nd}: \mathrm{YVO}_{4}$ laser (Millenia Xs, Spectra Physics). The pulse duration was about 2 ps (full width at half-maximum, fwhm), and the repetition rate of $81 \mathrm{MHz}$ of the excitation pulses could be decreased with a pulse picker unit (3980, Spectra Physics). In order to ensure homogeneous illumination conditions, we took care that the $1 / \mathrm{e}^{2}$-radius of the focused excitation light increased less than $2 \%$ over a region of $\pm 1 \mathrm{~mm}$ along the optical axis, which defined the focal volume for our experiments. Irreversible photobleaching was prevented by using a home-built rotating cuvette with an arm length of 9.5 $\mathrm{mm}$ that was spun at a frequency of $48 \mathrm{~Hz}$, thereby ensuring a constant flux of fresh RC-LH1 complexes. Fluorescence from the sample was collected under right angle geometry and spectrally filtered with a dielectric band-pass centered around $925 \mathrm{~nm}$ with a bandwidth of $40 \mathrm{~nm}$ (BP 925/40, AHF analysentechnik AG, Tübingen, Germany). The fluorescence emerging from the focal volume was imaged onto a streak camera system in combination with a CCD camera (C5680 and Orca-ER C4752, Hamamatsu Photonics). Due to the low quantum efficiency of the detector in the spectral region of the RC-LH1 emission, the data were not spectrally dispersed but reflected toward the detector via a mirror in the imaging spectrograph (250 IS, Bruker) that was installed in front of the streak system. Delay times introduced by the propagation of light along different paths from the sample toward the detector were corrected for using home-written software. For all experiments the detector system was operated in single sweep mode covering a time range of 2 ns resulting in an instrument response time of around $50 \mathrm{ps}$ (fwhm). In order to control the 


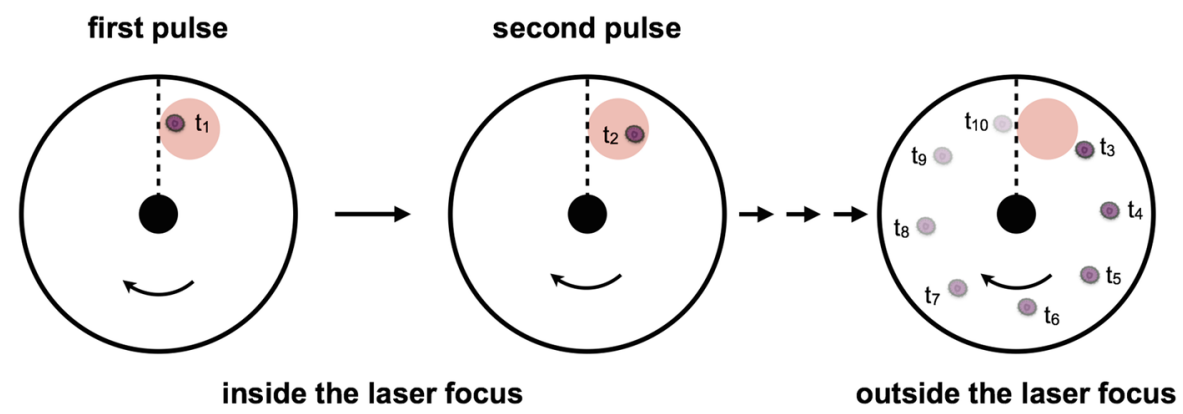

Figure 2. Pictorial representation of one revolution of the rotating cuvette for the example of a repetition rate of $81 \mathrm{kHz}$. The laser focus is indicated by the red shaded area, and the purple ellipses refer to the RC-LH1 complexes (not to scale). The complexes can be excited by multiple laser pulses during the rotation of the cuvette through the focus (here at $t_{1}$ and/or $t_{2}$ ) and evolve between the laser pulses as well as between transitions through the focus (here from $t_{3}$ until $t_{10}$ ). The decay of the excited states during the rotation of the cuvette is indicated by the fading color of the RC-LH1 complexes.

integrity of the RC-LH1 samples we recorded an absorption spectrum before and after the streak experiment using a commercial UV-vis spectrometer (Lambda 750, PerkinElmer). For none of the excitation conditions did we find significant bleaching or irreversible damage of the sample.

Experimental Protocol. The time-resolved fluorescence from RC-LH1 was recorded upon excitation at $879 \mathrm{~nm}$ as a function of the photon fluence (number of photons per pulse per area) using repetition rates of $81 \mathrm{MHz}, 8.1 \mathrm{MHz}, 810 \mathrm{kHz}$, and $81 \mathrm{kHz}$, respectively. For each repetition rate the photon fluence was varied in steps of $\sqrt{ } 10$ between $10^{10}$ (81 and 8.1 $\mathrm{MHz}), 10^{11}(810 \mathrm{kHz})$, or $10^{12}(81 \mathrm{kHz})$ photons $/\left(\right.$ pulse $\left.\cdot \mathrm{cm}^{2}\right)$, respectively, as lower limits and $10^{14}$ photons $/\left(\right.$ pulse $\left.\cdot \mathrm{cm}^{2}\right)$ for all repetition rates as the upper limit. These limits were determined either by the constraint to limit the integration time of the signal to less than $4 \mathrm{~h}$ or in order to stay just below the threshold where statistically more than one photon per pulse is absorbed by one RC-LH1 complex (given an absorption cross section of $1.43 \times 10^{-14} \mathrm{~cm}^{2}$ at $\left.879 \mathrm{~nm}^{64}\right)$. For the ease of comparison all transients are presented on a normalized scale such that 1 corresponds to the maximum of the respective transient and 0 to the averaged background level.

Computational Setup. For the simulation of the energy and electron transfer in isolated RC-LH1 complexes, we used a microstate description. ${ }^{65}$ We define a microstate as the tuple of a LH state and a RC state. For an empty LH1 ring the respective LH state alone corresponds to a microstate. More details will be outlined in the description of the model (see section Simulations). A master equation approach was chosen to compute the relative population of each individual microstate as a function of time. ${ }^{66}$

In order to mimic the rotating cuvette used in the experiments, the simulation was split into a part outside and a part inside the laser focus (see Figure 2).

Inside the laser focus, the excitation probability during each laser pulse was set to the product of the absorption crosssection $\left(1.43 \times 10^{-14} \mathrm{~cm}^{2}\right.$ at $\left.879 \mathrm{~nm}^{64}\right)$ and the excitation fluence. As we exclude the possibility of multiple excitations on one LH1 ring (see Model section for details), excitation probabilities exceeding one were set to unity. In accordance with the experimental conditions, the system was exposed to 2 , 21,208 , and 2080 consecutive pulses for repetition rates of 81 $\mathrm{kHz}, 810 \mathrm{kHz}, 8.1 \mathrm{MHz}$, and $81 \mathrm{MHz}$, respectively (for further details see Supporting Information). After excitation, each energy and electron transfer step was described by a transition between the respective microstates. Outside the laser focus, where no excitation occurs, the time evolution of the system was calculated by solving the master equation.

To obtain simulated fluorescence transients, we recorded the photon count rate as a function of time for all those states that contain a deexcitation from $\left|2_{L H}\right\rangle$ to $\left|1_{L H}\right\rangle$ or a deexcitation from $\left|4_{L H}\right\rangle$ to $\left|3_{L H}\right\rangle$ (vide infra). We obtained the total photon count rate $n_{f l u o}$ by summing the products of the occupation probability $P_{\nu}(t)$ of the corresponding fluorescent microstates with the respective fluorescence rate $k_{\nu}$ (eq 1 ).

$$
n_{\text {fluo }}(t)=\sum_{\nu=1}^{M} k_{\nu} P_{\nu}(t)
$$

To avoid an artifact caused by the initial phase of the simulations, we monitored the populations of the microstates of the system at the end of each revolution of the rotating cuvette. In the initial phase of the simulation, these populations vary strongly as a function of the number of revolutions that have already passed, while in a later phase these populations converge toward fixed values (see Supporting Information Figure S2). For the reported simulated fluorescence transients, we only considered the revolution after convergence of the populations and discarded those from the initial phase.

\section{RESULTS AND DISCUSSION}

The fluorescence decays of isolated RC-LH1 complexes were registered as a function of both the photon fluence and the repetition rate of the laser. The observed transients are displayed in Figure 3 (black dots) together with the results from master equation simulations that will be discussed later. The data in each column of the figure correspond to a distinct repetition rate that decreases from left to right. Within each column the photon fluence (given in units of photons/(pulse. $\left.\mathrm{cm}^{2}\right)$ ) increases from top to bottom. The transients are arranged such that within each row the continuous wave $(\mathrm{cw})$ equivalent of the excitation intensity (number of photons/ (time-area)) is constant; i.e., the product of the photon fluence and the repetition rate is the same along a row.

As a starting point the transients were fitted as a superposition of three exponential decays. For a fixed repetition rate the decay times were treated as global parameters, and only the amplitudes of the three contributions were allowed to vary. The general picture that emerges from this approach is as follows: At low fluences the transients can be described as biexponentials ( $40 \mathrm{ps}, 600 \mathrm{ps}$ ), at intermediate fluences as triexponentials (40 ps, $200 \mathrm{ps}, 600 \mathrm{ps}$ ), and at high fluences 


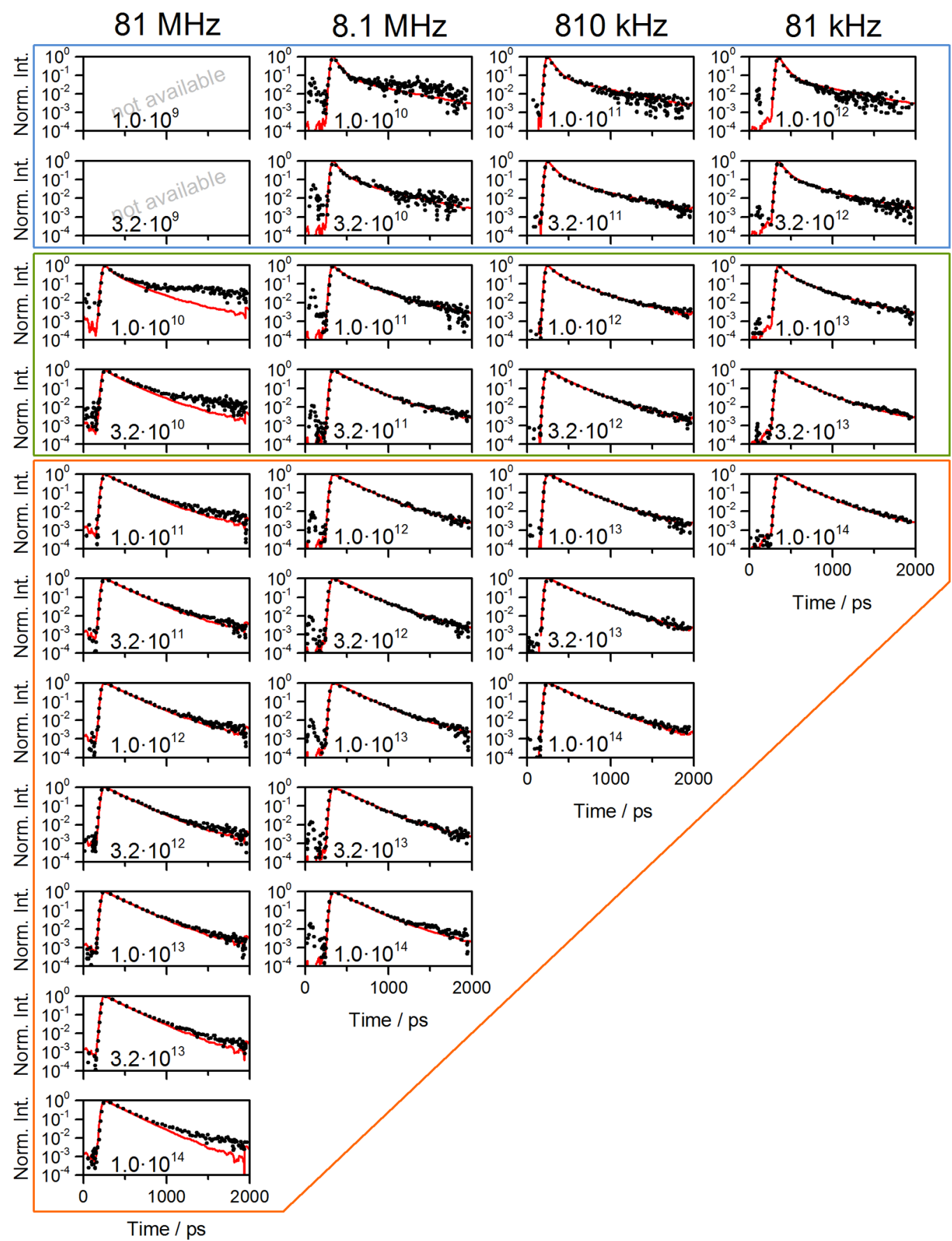

Figure 3. Normalized fluorescence decays (black dots) of isolated RC-LH1 complexes in detergent solution as a function of the repetition rate (columns) and the photon fluence (rows) of the excitation together with simulated fluorescence decays (red lines). The repetition rate decreases from left to right, and the photon fluence which is given in units of photons $/\left(\right.$ pulse $\left.\cdot \mathrm{cm}^{2}\right)$ within each graph increases from top to bottom. Within each row the $\mathrm{cw}$ equivalent excitation intensity is constant. The colored boxes indicate the range of excitation parameters for which we find from the simulations that the majority of RC-LH1 complexes is in an open state (more than $81 \%$ open RCs, blue box), where RCs in the open and closed state coexist (20-80\% open RCs, green box) and where RCs in the closed state dominate (below $20 \%$ open RCs, orange box).

again as biexponentials (200 ps, 600 ps). Thereby, the amplitude of the 200 ps component grows for increasing fluences, whereas that of the 40 ps component decreases at the same time, until it becomes undetectably small at the highest fluence applied. The amplitude of the slow component does not depend significantly on the fluence. It is interesting to note that within experimental error the transients that were measured with the same equivalent $\mathrm{cw}$ excitation intensity yield identical results with the fits. Deviations of the amplitudes from this schedule are only observable for the highest repetition rate of $81 \mathrm{MHz}$. At that rate we find for both, the lowest and the highest fluence, that the amplitude of the slowest decay component is enhanced with respect to what has been found at the other repetition rates.

Upon electronic excitation of the special pair a sequence of electron transfers is initiated leaving behind a positively charged special pair $\mathrm{P}^{+}$. For excitation rates that are higher than the inverse of the recovery time of the $\mathrm{RC}$, which amounts to about 
$100 \mathrm{~ms}$, it is known that the sample accumulates a mixture of RC-LH1 complexes with open (P) and closed $\left(\mathrm{P}^{+}\right) \mathrm{RCs}$ after a few excitation cycles. ${ }^{52-55,60,67}$ Therefore, we tentatively attribute the 40 and 200 ps components to reflect the fractions of RCs in the open and closed state, respectively, which is in qualitative agreement with the changes of the corresponding amplitudes as a function of the excitation rate. Moreover, since the amplitude of the slow component does not depend significantly on the excitation rate, it is possible to associate the 600 ps component with a residual population of "empty" LH1 rings that might reflect a consequence of the extraction procedure. This conjecture is in line with the fact that the observed lifetime is in close agreement with the 650 ps decay time that has been found for chromatophores of a mutant without RCs. 57

In order to test the influence of the redox state of the RC on the dynamics of the fluorescence decays we performed two control experiments. For the first one the RC was chemically reduced to shift the steady-state population of the RCs to the open state P. This experiment had to be conducted on a newly prepared batch of RC-LH1 complexes that were grown and purified following the same protocol as before. For these complexes the fraction of the long-lived component was enhanced with respect to the previous sample. The general variation of the populations as a function of the excitation conditions, however, was the same as described above. This sample was illuminated with a fluence of $10^{14}$ photons/(pulse. $\mathrm{cm}^{2}$ ) at a repetition rate of $810 \mathrm{kHz}$, while the reducing agent $\mathrm{DAD}$ was added to the sample up to a final concentration of 1 $\mathrm{mM}$. Examples of the corresponding fluorescence decays are shown in Figure 4 for $\mathrm{DAD}$ concentrations of $0 \mu \mathrm{M}$ (orange

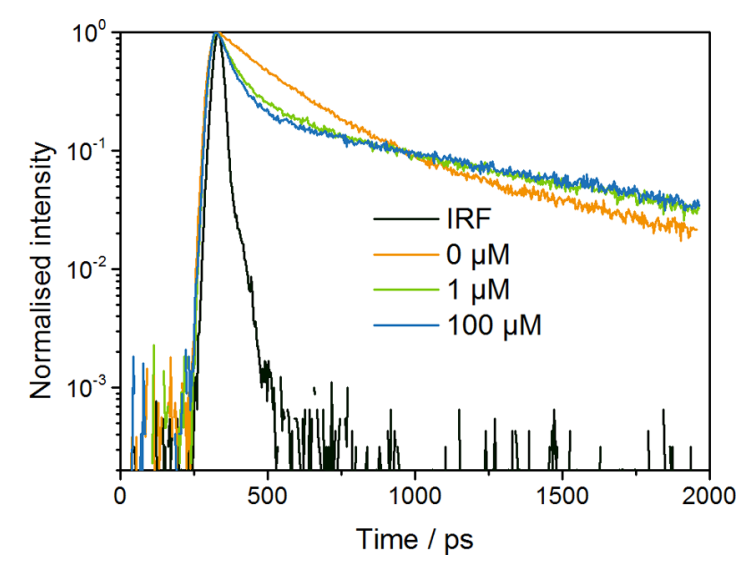

Figure 4. Normalized fluorescence decays of isolated RC-LH1 complexes in detergent solution for a repetition rate of $810 \mathrm{kHz}$ and a fluence of $10^{14}$ photons $/\left(\right.$ pulse $\left.\cdot \mathrm{cm}^{2}\right)$ as a function of the diaminodurene (DAD) concentration (colored curves). The black curve refers to the instrument response and has a width of $50 \mathrm{ps}$ (fwhm).

line), $1 \mu \mathrm{M}$ (green line), and $100 \mu \mathrm{M}$ (blue line). The transient recorded in the absence of any reducing agent shows a biexponential decay with time constants of 200 and 600 ps (Figure 4 orange line). Upon increasing the concentration of $\mathrm{DAD}$ a further decay component with a time constant of $40 \mathrm{ps}$ grows in, whereas at the same time the amplitude of the $200 \mathrm{ps}$ component decreases and finally vanishes at a DAD concentration of $100 \mu \mathrm{M}$. For even higher DAD concentrations no further changes of the transients could be detected. During the whole sequence of experiments the amplitude of the 600 ps component did not change significantly.

For the second control experiment the RC was chemically oxidized and the steady-state population of the RCs was shifted toward the closed state $\mathrm{P}^{+}$. The sample was illuminated with a fluence of $3.2 \times 10^{10}$ photons $/\left(\right.$ pulse $\left.\cdot \mathrm{cm}^{2}\right)$ at a repetition rate of $8.1 \mathrm{MHz}$, while the oxidizing agent $\mathrm{K}_{3} \mathrm{Fe}(\mathrm{CN})_{6}$ was added to the sample up to a final concentration of $1 \mathrm{mM}$. Examples of the corresponding fluorescence decays are shown in Figure 5

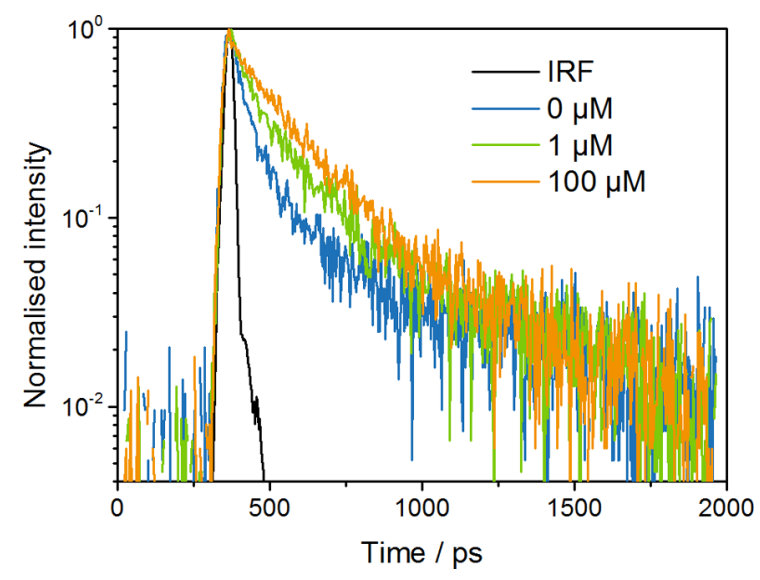

Figure 5. Normalized fluorescence decays of isolated RC-LH1 complexes in detergent solution for a repetition rate of $8.1 \mathrm{MHz}$ and a fluence of $3.2 \times 10^{10}$ photons $/\left(\right.$ pulse $\left.\cdot \mathrm{cm}^{2}\right)$ as a function of the potassium hexacyanoferrate(III) $\left(\mathrm{K}_{3} \mathrm{Fe}(\mathrm{CN})_{6}\right)$ concentration (colored curves). The black curve refers to the instrument response and has a width of 50 ps (fwhm).

for oxidant concentrations of $0 \mu \mathrm{M}$ (blue line), $1 \mu \mathrm{M}$ (green line), and $100 \mu \mathrm{M}$ (orange line). The transient recorded in the absence of the oxidizing agent (Figure 5, blue line) shows a triexponential decay with time constants of 40 ps, 200 ps, and 600 ps, where the amplitude of the 200 ps component is very small. Adding the oxidant results in an increase of the amplitude of the $200 \mathrm{ps}$ component at the expense of the amplitude of the 40 ps component (Figure 5, green line) until the latter becomes undetectable at a concentration of $100 \mu \mathrm{M}$. As before the amplitude of the $600 \mathrm{ps}$ component does not change significantly as a function of the $\mathrm{K}_{3} \mathrm{Fe}(\mathrm{CN})_{6}$ concentration. We note that we observe a strong quenching of the RC-LH1 fluorescence as well as a bleaching of the B875 absorption band for $\mathrm{K}_{3} \mathrm{Fe}(\mathrm{CN})_{6}$ concentrations above $100 \mu \mathrm{M}$, which is likely to be caused by an oxidation and concomitant destruction of the LH1 ring as has been found before. ${ }^{68}$

Apparently, the increase of the concentration of the reducing agent, i.e., shifting the steady state population of the RCs to the neutral state $\mathrm{P}$ ( $\mathrm{RC}$ open), has qualitatively the same effect (increase of the 40 ps component; decrease of the 200 ps component) as decreasing the excitation rate for an unmanipulated sample. Vice versa, increasing the concentration of the oxidant, i.e., shifting the steady-state populations of the special pairs toward the $\mathrm{P}^{+}$state (RC closed), has the same effect (decrease of the 40 ps component; increase of the 200 ps component) as increasing the excitation rate for an unmanipulated sample. These similarities support the association of the three observed decay components with pigmentprotein complexes that have the $\mathrm{RC}$ in the open state (40 ps) or in the closed state (200 ps) and those that feature an "empty" LH1 ring (600 ps). 
Simulations. Model. The DDM concentration in our samples is about $0.4 \mathrm{mM}$, which is above the critical micellar concentration of $0.17 \mathrm{mM}$ at $25{ }^{\circ} \mathrm{C}$ for this detergent. Therefore, we consider the sample to consist of nonaggregated RC-LH1 complexes that do not interact with each other, and we exclude intercomplex energy transfer. In order to obtain a more quantitative understanding of the processes that take place in the RC-LH1 complexes upon photoexcitation we performed simulations based on a master equation approach. The computational details are explained in the Materials and Methods section.

Empty $L H 1$. As a starting point for the modeling, we resort to the same concept that has been used to obtain a quantitative description of the dynamics of the photophysical processes in nonaggregated $\mathrm{LH} 2$ complexes. ${ }^{62}$ The basic idea of this approach was to treat the $\mathrm{LH} 2$ as a multichromophoric system that can carry more than one electronic excitation at the same time. Because there was no need to specify the exact size of the assembly, we will use an equivalent approach for modeling the fraction of "empty" LH1 rings.

In Figure 6, we use a pictorial representation of the electronic states of a nonaggregated LH1 ring that has been proven to be
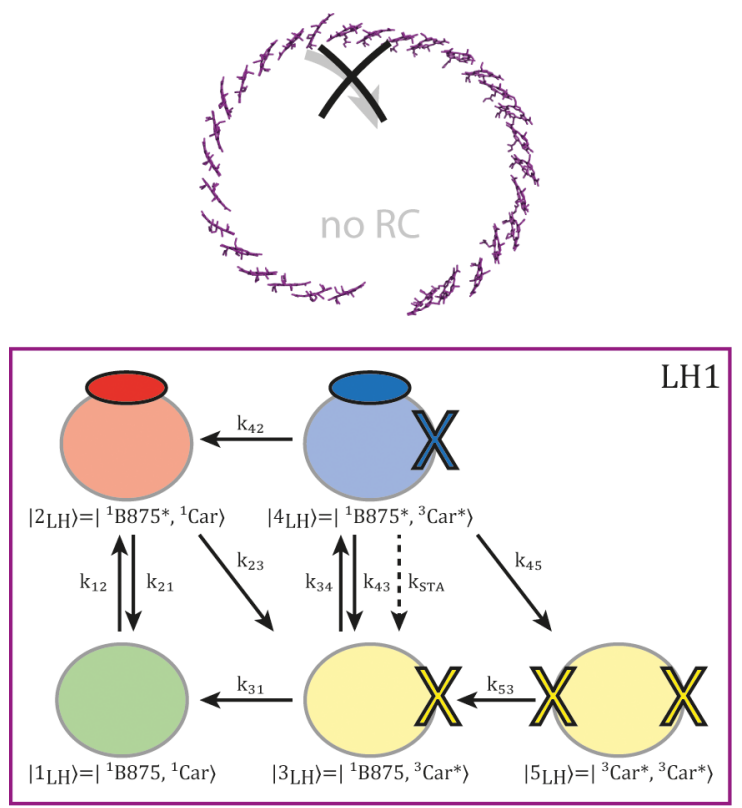

Figure 6. Pictorial representation of the transitions between the electronic states of an isolated LH1 ring without RC. The colored spheres represent the B875 pigment pool of LH1 in different electronic states, where ellipses correspond to singlet excitations and crosses to triplet excitations. Singlet-triplet annihilation (STA) is taken into account. For more details see text.

very useful in the past. ${ }^{6,63}$ The LH1 ring in the various states is represented by colored spheres that are connected by transitions that have specific rates $k_{i j}$. A LH1 ring in the electronic ground state is represented by a green sphere. For brevity, we refer to this state as $\left|1_{L H}\right\rangle=\mid{ }^{1} B 875,{ }^{1}$ Car $\rangle$. Upon excitation into the $\mathrm{B} 875$ band, there will be a fast equilibration within the exciton states, which is beyond the time resolution of our experiment, and we therefore treat the ${ }^{1} \mathrm{~B} 875^{*}$ excited state as an effective level. In Figure 6 this state is visualized by a red sphere with an ellipse and is referred to as $\left|2_{L H}\right\rangle=\mid{ }^{1} B 875^{*}$, ${ }^{1}$ Car . Under high-illumination conditions, it becomes possible to create two excited ${ }^{1} \mathrm{~B} 875^{*}$ states in the same RC-LH1 complex, giving rise to singlet-singlet annihilation (SSA). In earlier studies on RC-LH1-only chromatophores or LH2 samples of $\mathrm{mM}$ concentrations, it has been found that fluences above $3 \times 10^{14}$ photons $/\left(\right.$ pulse $\left.\cdot \mathrm{cm}^{2}\right)$ are required to have more than one singlet state on an individual light-harvesting complex. ${ }^{69,70}$ However, in those samples the possible intercomplex energy transfer increases the probability by orders of magnitude so that a $\mathrm{LH}$ complex receives a second excitation. ${ }^{63}$ Although the highest fluence used in our work is close to the threshold given above, we rule out the possibility for SSA, because we work at $\mathrm{nM}$ concentrations, which excludes populating the $\left|2_{\mathrm{LH}}\right\rangle$ state by intercomplex energy transfer. From the $\left|2_{\mathrm{LH}}\right\rangle$ state the system can decay back to the ground state or cross over within 10 ns to the triplet state $\left.{ }^{3} \mathrm{~B} 875^{*},{ }^{1} \mathrm{Car}\right\rangle$ which is quenched by the carotenoids with a rate of $(10 \mathrm{~ns})^{-171}$ resulting in the state $\left|3_{L H}\right\rangle=\left|{ }^{1} B 875,{ }^{3} \mathrm{Car} *\right\rangle$. This state is displayed in Figure 6 as a yellow sphere with a cross that symbolizes a LH1 ring that carries a triplet state on one of the carotenoids. In what follows the transition from $\left|2_{L H}\right\rangle$ to $\left|3_{L H}\right\rangle$ will be described by an effective rate that amounts to $(20 \mathrm{~ns})^{-1}$. In RC-LH1 complexes that contain spirilloxanthin as carotenoids the subsequent decay of the carotenoid triplet state occurs with a time constant between 1.6 and $2.9 \mu \mathrm{s} .^{72,73}$ Singlet-triplet annihilation (STA) becomes possible if a LH1 ring that still carries a triplet state (cross) gets excited again to the ${ }^{1} \mathrm{~B} 875^{*}$ state (ellipse). This situation is depicted by a circle with both a cross and an ellipse and will be referred to as $\left|4_{L H}\right\rangle$ $=\left|{ }^{1} B 875^{*},{ }^{3} \mathrm{Car}^{*}\right\rangle$, Figure 6, blue sphere. However, since LH1 is a multichromophoric system, it is also possible that a LH1 ring that is in state $\left|4_{\mathrm{LH}}\right\rangle$ decays with a rate $k_{45}$ to a state $\left|S_{L H}\right\rangle=$ $\left.{ }^{3} \mathrm{Car}^{*},{ }^{3} \mathrm{Car}^{*}\right\rangle$ having two triplet states on different carotenoids. Since it is known from ESR experiments that the triplet states on the carotenoids are immobile ${ }^{74}$ and because triplet-triplet energy transfer is mediated by the short-range exchange interaction, we neglect triplet-triplet annihilation in our analysis. However, for state $\left|4_{\mathrm{LH}}\right\rangle$ we do take into account the additional decay channel to state $\left|S_{\mathrm{LH}}\right\rangle$. The numerical values for the rates that are required for a quantitative modeling have been taken from the literature, the current study, or the previous study on LH2 and are summarized in Table 1 together with the corresponding references.

Table 1. Input Parameters for the Simulations That Are Associated with LH1

\begin{tabular}{|c|c|c|c|}
\hline \multicolumn{4}{|c|}{ LH1 } \\
\hline parameter & type & value & reference \\
\hline$\sigma_{\mathrm{B} 875}$ & $\begin{array}{l}\text { absorption cross section } \\
{ }^{1} \mathrm{~B} 875\end{array}$ & $1.43 \times 10^{-14} \mathrm{~cm}^{2}$ & 64 \\
\hline$k_{12}$ & excitation rate ${ }^{1} \mathrm{~B} 875$ & $\sigma_{\mathrm{B} 875} \cdot J_{\mathrm{ex}}$ & \\
\hline$k_{21}$ & singlet decay ${ }^{1} \mathrm{~B} 875^{*}$ & $(600 \mathrm{ps})^{-1}$ & this work \\
\hline$k_{23}$ & intersystem crossing to ${ }^{3} \mathrm{Car}^{*}$ & $(20 \mathrm{~ns})^{-1}$ & 71 \\
\hline$k_{31}$ & triplet decay ${ }^{3} \mathrm{Car}^{*}$ & $(1.6 \mu \mathrm{s})^{-1}$ & 72 \\
\hline$k_{34}$ & excitation rate ${ }^{1} \mathrm{~B} 875$ & $=k_{12}$ & \\
\hline$k_{43}$ & singlet decay ${ }^{1} \mathrm{~B} 875^{*}$ & $=k_{21}$ & this work \\
\hline$k_{\mathrm{STA}}$ & singlet triplet annihilation & $(5.56 \mathrm{~ns})^{-1}$ & 62 \\
\hline$k_{42}$ & triplet decay ${ }^{3}$ Car* & $=k_{31}$ & \\
\hline$k_{45}$ & intersystem crossing to ${ }^{3} \mathrm{Car} *$ & $=k_{23}$ & \\
\hline$k_{53}$ & triplet decay ${ }^{3}$ Car* & $=2 \cdot k_{31}$ & \\
\hline$k_{\text {open }}$ & transfer ${ }^{1} \mathrm{~B} 875^{*}$ to $\mathrm{P}$ & $(40 \mathrm{ps})^{-1}$ & this work \\
\hline$k_{\text {back }}$ & transfer $\mathrm{P}^{*}$ to ${ }^{1} \mathrm{~B} 875$ & $(8 \mathrm{ps})^{-1}$ & 54 \\
\hline$k_{\text {closed }}$ & transfer ${ }^{1} \mathrm{~B} 875^{*}$ to $\mathrm{P}^{+}$ & $(300 \mathrm{ps})^{-1}$ & this work \\
\hline
\end{tabular}



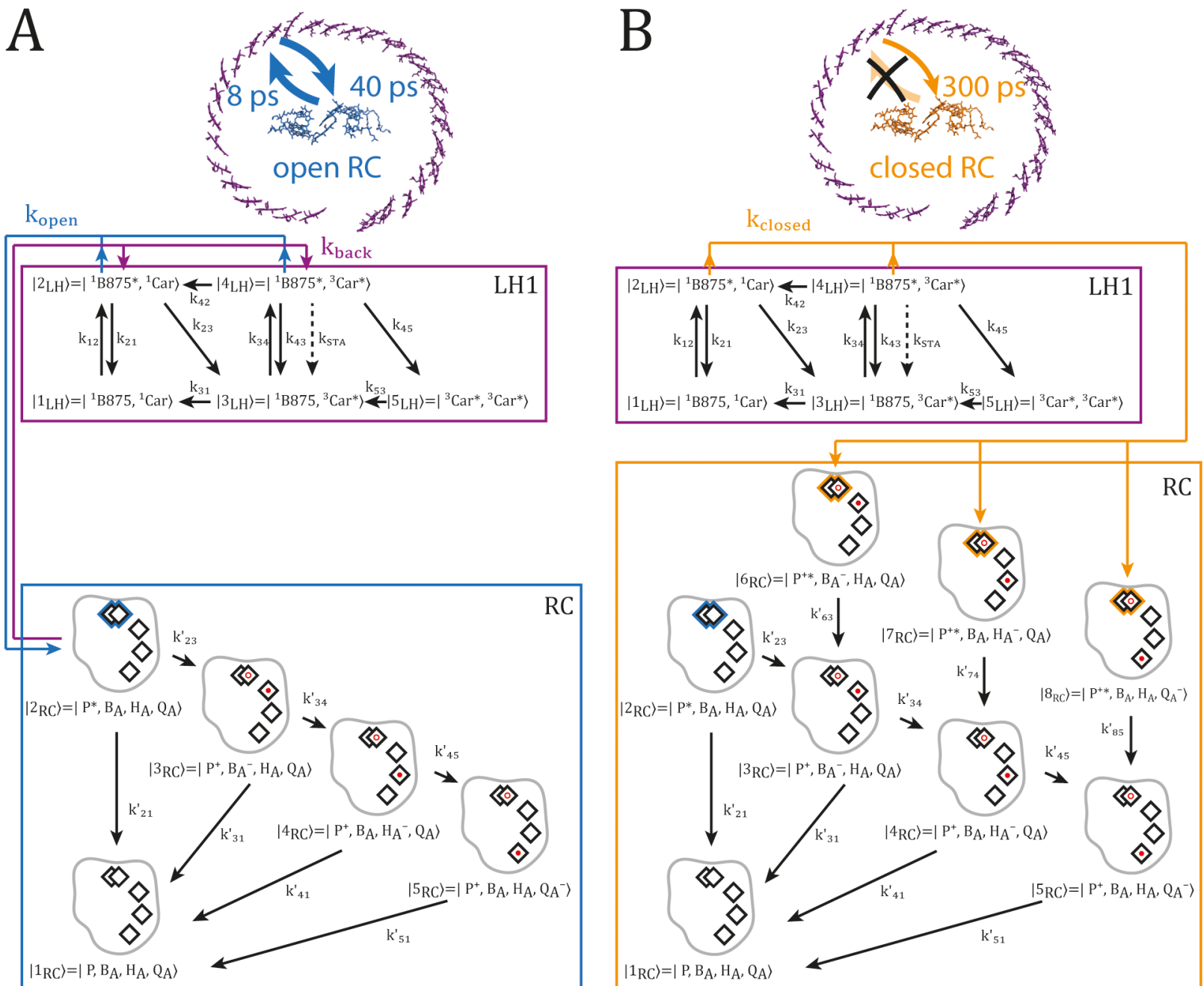

Figure 7. Pictorial representation of the energy and electron transfer dynamics in RC-LH1 complexes with open (A) or closed (B) RCs. The top boxes refer to the description of empty LH1 rings as in Figure 6. The lower boxes refer to the description of the RCs. In the RC, the cofactors are shown as diamonds for one branch only. An electronic excitation is indicated by a colored halo, and oxidized (reduced) states are represented by an open (closed) dot.

Open RC. For modeling the RC-LH1 complexes that contain a $\mathrm{RC}$ we start the description with those complexes that feature an open RC. In addition to the processes that occur within the LH1 ring and which have been described above, we have to take into account the photophysical and photochemical processes that take place in the RC after the photoexcitation. In order to avoid confusion, electronic states that are associated with the LH1 ring are indexed as "LH", whereas those that are associated with the RC are indexed as "RC". For the sake of brevity, the RC is depicted at the bottom of Figure 7 with the pigments of the A-branch (diamonds), only. We do not consider direct excitation of the special pair by the laser because of its low absorption cross section with respect to that of the B875 pigment pool at the wavelength used. In our nomenclature, transfer of excitation energy from the LH1 ring to the RC can occur from the states $\left|2_{L H}\right\rangle$ and $\left|4_{L H}\right\rangle$. The time constant for this processes is $40 \mathrm{ps}$ and induces the transition of the $\mathrm{RC}$ from the ground state $\left|1_{\mathrm{RC}}\right\rangle=\mid \mathrm{P}, \mathrm{B}_{\mathrm{A}}, \mathrm{H}_{\mathrm{A}}$ $\left.\mathrm{Q}_{\mathrm{A}}\right\rangle$ to a state with the special pair in the excited state $\left|2_{\mathrm{RC}}\right\rangle=$ $\left|\mathrm{P}^{*}, \mathrm{~B}_{\mathrm{A}}, \mathrm{H}_{\mathrm{A}}, \mathrm{Q}_{\mathrm{A}}\right\rangle$ (see Figure $7 \mathrm{~A}$, blue box). Back transfer from state $\left|2_{R C}\right\rangle$ to the LH1 has been observed and occurs with a time constant of 8 ps. ${ }^{46,47,54}$ Alternatively, the excitation of the special pair initiates an electron transfer chain via the accessory
BChl $\mathrm{B}_{\mathrm{A}}\left(\left|3_{\mathrm{RC}}\right\rangle=\left|\mathrm{P}^{+}, \mathrm{B}_{\mathrm{A}}^{-}, \mathrm{H}_{\mathrm{A}}, \mathrm{Q}_{\mathrm{A}}\right\rangle ; 3 \mathrm{ps}\right)$ and the $\mathrm{BPhe} \mathrm{H}_{\mathrm{A}}$ $\left(\left|4_{\mathrm{RC}}\right\rangle=\left|\mathrm{P}^{+}, \mathrm{B}_{\mathrm{A}}, \mathrm{H}_{\mathrm{A}}^{-}, \mathrm{Q}_{\mathrm{A}}\right\rangle ; 0.9 \mathrm{ps}\right)$ to a ubiquinone molecule $\mathrm{Q}_{\mathrm{A}}$ $\left(\left|5_{\mathrm{RC}}\right\rangle=\left|\mathrm{P}^{+}, \mathrm{B}_{\mathrm{A}}, \mathrm{H}_{\mathrm{A}}, \mathrm{Q}_{\mathrm{A}}^{-}\right\rangle ; 200 \mathrm{ps}\right)$, where the charge-separated state is stabilized. ${ }^{32}$ In vivo the charge stabilized on $\mathrm{Q}_{A}^{-}$would be transferred within about $100 \mu \mathrm{s}^{75}$ to a second ubiquinone molecule $\mathrm{Q}_{\mathrm{B}}$. This molecule is loosely bound to the $\mathrm{RC}$ protein matrix and often lost during the purification process. From test simulations we learned that the presence (or lack) of $\mathrm{Q}_{\mathrm{B}}$ in the RCs has only a minor influence on the fluorescence transients (see Supporting Information, Figure S3) and was therefore neglected in this study. Relaxation from the states $\left|2_{R C}\right\rangle,\left|3_{R C}\right\rangle$, $\left|4_{R C}\right\rangle$, and $\left|5_{R C}\right\rangle$ to the ground state $\left|1_{R C}\right\rangle$ occurs with time constants of 180 ps, 200 ps, $10 \mathrm{~ns}$, and $100 \mathrm{~ms}$, respectively. ${ }^{76-78}$ The rates of the transitions between the five states associated with the RC are denoted with $k_{i j}{ }^{\prime}$ in the blue box in Figure 7A and are summarized in Table 2. We have also neglected the transition of the radical pair ${ }^{1}\left(\mathrm{P}^{+}, \mathrm{H}_{\mathrm{A}}^{-}\right)$to the triplet state ${ }^{3}\left(\mathrm{P}^{+}, \mathrm{H}_{\mathrm{A}}^{-}\right)$, because this conversion occurs only in $\mathrm{RCs}$ without $\mathrm{Q}_{\mathrm{A}}$ and at very high illumination intensities, which are both not the case here.

Closed RC. First off, it is important to note that the definition of a closed RC is not uniform in the literature. Here, we refer to the $\mathrm{RC}$ as being in the closed state if the special pair 
Table 2. Input Parameters for the Simulations That Are Associated with the RC

\begin{tabular}{clll}
\multicolumn{2}{c}{$\mathrm{RC}$} & & \multicolumn{1}{c}{ type } \\
\hline parameter & \multicolumn{1}{c}{ value } & reference \\
$k_{21}{ }^{\prime}$ & singlet decay $\mathrm{P}^{*}$ & $(180 \mathrm{ps})^{-1}$ & 77 \\
$k_{23}{ }^{\prime}$ & charge separation to $\left(\mathrm{P}^{+}, \mathrm{B}_{\mathrm{A}}^{-}\right)$ & $(3 \mathrm{ps})^{-1}$ & 32 \\
$k_{31}{ }^{\prime}$ & charge recombination to $\mathrm{P}$ & $(200 \mathrm{ps})^{-1}$ & 78 \\
$k_{34}{ }^{\prime}$ & charge separation to $\left(\mathrm{P}^{+}, \mathrm{H}_{\mathrm{A}}^{-}\right)$ & $(0.9 \mathrm{ps})^{-1}$ & 32 \\
$k_{41}{ }^{\prime}$ & charge recombination to $\mathrm{P}$ & $(10 \mathrm{~ns})^{-1}$ & 76 \\
$k_{45}{ }^{\prime}$ & charge separation to $\left(\mathrm{P}^{+}, \mathrm{Q}_{\mathrm{A}}^{-}\right)$ & $(200 \mathrm{ps})^{-1}$ & 32 \\
$k_{51}{ }^{\prime}$ & charge recombination to $\mathrm{P}$ & $(100 \mathrm{~ms})^{-1}$ & 76 \\
$k_{63}{ }^{\prime}$ & singlet decay $\mathrm{P}^{+*}$ & $\infty$ & see text \\
$k_{74}{ }^{\prime}$ & singlet decay $\mathrm{P}^{+*}$ & $\infty$ & see text \\
$k_{85}{ }^{\prime}$ & singlet decay $\mathrm{P}^{+*}$ & $\infty$ & see text \\
\hline
\end{tabular}

is in the oxidized state $\mathrm{P}^{+}$. As has been demonstrated before, the $\mathrm{P}^{+}$state can still act as an acceptor for the excitation energy from $\mathrm{LH} 1$, yet at a reduced energy transfer rate. ${ }^{54,55,79}$ For the description of RC-LH1 complexes with a closed RC, we resort to the details described above and extend the RC model by three states. These correspond to a situation where $\mathrm{P}$ is oxidized but resides in an excited state $\mathrm{P}^{+*}$, and either $\mathrm{B}_{\mathrm{A}}, \mathrm{H}_{\mathrm{A}}$ or $\mathrm{Q}_{\mathrm{A}}$ is in the reduced state. These states are denoted as $\left|6_{\mathrm{RC}}\right\rangle$ $=\left|\mathrm{P}^{+*}, \mathrm{~B}_{\mathrm{A}}^{-}, \mathrm{H}_{\mathrm{A}}, \mathrm{Q}_{\mathrm{A}}\right\rangle,\left|7_{\mathrm{RC}}\right\rangle=\left|\mathrm{P}^{+*}, \mathrm{~B}_{\mathrm{A}}, \mathrm{H}_{\mathrm{A}}^{-}, \mathrm{Q}_{\mathrm{A}}\right\rangle$ and $\left|8_{\mathrm{RC}}\right\rangle=\mid \mathrm{P}^{+*}$, $\left.B_{A}, H_{A}, Q_{A}^{-}\right\rangle$(see Figure $7 B$, orange box), and the corresponding energy transfer rates from $\left|2_{L H}\right\rangle$ or $\left|4_{L H}\right\rangle$ to either of these states are referred to as $k_{\text {closed. }}$. A numerical value for this rate can be obtained from the measured decay time of $200 \mathrm{ps}$ for this case. Using $k_{\text {meas }}=(200 \mathrm{ps})^{-1}=k_{21}+k_{\text {closed }}+k_{23}$ $+k_{\text {STA }} \approx k_{21}+k_{\text {closed }}$ and $k_{21}=(600 \mathrm{ps})^{-1}$ yields $k_{\text {closed }}=(300$ $\mathrm{ps})^{-1}$. Unfortunately, little is known about the exact decay process of the $\mathrm{P}^{+*}$ state, yet it is conceivable that the relaxation from $\mathrm{P}^{+*}$ to the $\mathrm{P}^{+}$ground state is beyond the temporal resolution of our experiment. ${ }^{79}$ Therefore, we have modeled the transitions from $\left|6_{R C}\right\rangle$ to $\left|3_{R C}\right\rangle$, from $\left|7_{R C}\right\rangle$ to $\left|4_{R C}\right\rangle$, and from $\left|8_{R C}\right\rangle$ to $\left|5_{R C}\right\rangle$ as instantaneous and do not consider back transfer of the excitation to the LH1 ring from these states. The rates used for the simulations for a closed RC are also summarized in Table 2.

Further Parameters. In addition to the parameters summarized in the tables, we have to specify the fraction of pigment-protein complexes that carry no RC, as well as the fraction of RC-LH1 complexes that freshly enter the laser focus during the revolution of the rotating cuvette that contains the sample. The first parameter can be estimated from the relative amplitude of the slow decay component, and we find satisfactory agreement between the simulations and the whole data set of 30 transients only if this value is kept in a range of ( 3 \pm 2 )\% (see Supporting Information Figure S4). The fraction of fresh sample that enters the focus was estimated to be $25 \%$. This parameter can be varied between $25 \%$ and $50 \%$ yielding minor but visible deviations between the measured and simulated decay curves (see Supporting Information Figure S5). The remaining fraction of RC-LH1 complexes was treated as having been already exposed to the laser during the previous passage. All transients were then simulated simultaneously using a Master equation approach (see also Materials and Methods section).

Results of the Simulations. The simulated curves that were obtained using the formalism described above are shown in Figure 3 as red lines. The overall agreement between the measured and the simulated transients is striking, in particular, because no free parameters for fitting of individual decays have been used. Rather we applied one global model to describe all the curves simultaneously. For the lowest three repetition rates, $81 \mathrm{kHz}, 810 \mathrm{kHz}$, and $8.1 \mathrm{MHz}$, there is only a small but observable deviation of the simulated decay from the experimentally observed one for the lowest fluence at 8.1 $\mathrm{MHz}$. For this parameter setting, the photon counting rate (and concomitantly the signal-to-noise ratio) is rather low, which might cause an artifact when the transient is normalized for comparison. At the highest repetition rate of $81 \mathrm{MHz}$ a very good agreement between simulation and experiment can be found but only for fluences between $10^{11}$ photons $/\left(\right.$ pulse $\left.\cdot \mathrm{cm}^{2}\right)$ and $3.2 \times 10^{13}$ photons $/\left(\right.$ pulse $\left.\cdot \mathrm{cm}^{2}\right)$. For the two lowest fluences, the simulated transients show a significantly faster decay than the experimentally observed ones, and also for the highest fluence, an observable albeit not dramatic difference between simulation and experiment is apparent at longer times. These discrepancies reflect a stronger contribution of the fraction of LH1 without RC at the lowest and highest fluences, when the time interval between two succeeding laser pulses decreases from about 120 to 12 ns. On first sight this observation seems to indicate the involvement of long-lived triplet states and concomitant STA. However, this process is already included in the simulations, and we find a nearly perfect match between experiment and simulation at the other repetition rates where STA is also relevant. In order to find another explanation for the deviations between the simulated and experimentally observed transients at $81 \mathrm{MHz}$, we performed further test simulations. First we adjusted the fraction of complexes without RC such that simulation and experiment matched for $10^{10}$ photons/(pulse $\left.\cdot \mathrm{cm}^{2}\right)$ at $81 \mathrm{MHz}$. However, this resulted in a strong mismatch between the calculated and observed transients at all other repetition rates. Second we considered the possibility of SSA, which was not taken into account for the simulations. Since for the highest repetition rate of $81 \mathrm{MHz}$ the temporal separation between two consecutive laser pulses already exceeds the fluorescence lifetime by more than a factor of 10 this requires the creation of two ${ }^{1} \mathrm{~B} 875^{*}$ excitations within a single laser pulse. This effect then should solely depend on the fluence and not on the applied repetition rate. However, such a deviation between experiment and simulation is not observable at the highest fluences at repetition rates other than $81 \mathrm{MHz}$, which excludes this option as well. Nevertheless, for most of the combinations of fluence and repetition rate the model used for the simulations predicts the dynamics of RC-LH1 that are in excellent agreement with the experiments. Therefore, we used this model to extract the relative number of LH1 complexes that carry a triplet state as well as the fraction of RC-LH1 complexes with a closed $\mathrm{RC}$ as a function of the excitation parameters.

The relative steady-state triplet population (the relative number of RC-LH1 complexes that carry at least one triplet state) is shown in Figure 8A as a function of the fluence for the applied repetition rates. Expectedly, this fraction is zero for all fluences at the repetition rate of $81 \mathrm{kHz}$ as the time delay between two successive pulses of about $12 \mu$ s exceeds the lifetime of the triplet state by at least a factor of 5. For the other three repetition rates the fraction of RC-LH1 complexes that carry ${ }^{3} \mathrm{Car}^{*}$ states on the LH1 ring increases by several orders of magnitude with increasing fluence; see Figure 8A. Representing the same data as a function of the repetition rate for the $\mathrm{cw}$ equivalent excitation intensities, Figure $8 \mathrm{~B}$, 

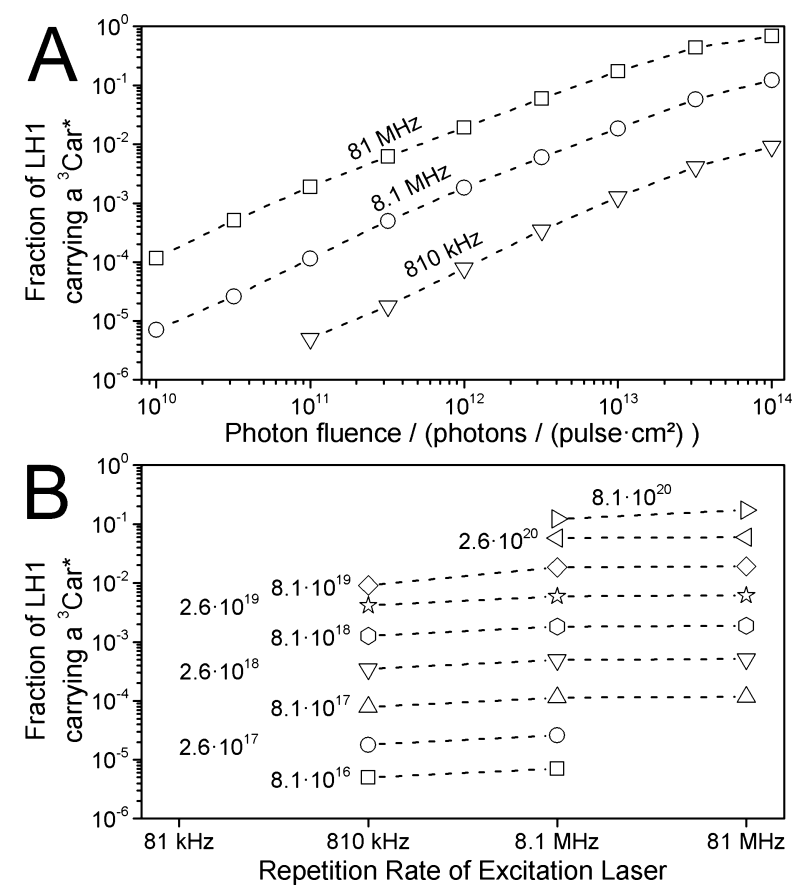

Figure 8. Simulated fraction of RC-LH1 complexes that carry at least one triplet excitation on the LH1 ring $\left({ }^{3} \mathrm{Car}^{*}\right)(\mathrm{A})$ as a function of the fluence and (B) as a function of the repetition rate. The lines serve as a guide for the eye and connect data points of equal repetition rate (A) or equal $\mathrm{cw}$ equivalent excitation intensity (B), given in photons/(s. $\mathrm{cm}^{2}$ ). Conversions into other dimensions are provided in the Supporting Information, Table S2.

reveals for all intensities an increase of the triplet population for rising the repetition rate from $810 \mathrm{kHz}$ to $8.1 \mathrm{MHz}$, which levels off for a further increase of the repetition to $81 \mathrm{MHz}$. Apparently the pulse separation at $8.1 \mathrm{MHz}$ of $120 \mathrm{~ns}$ is already so much faster than the triplet decay time of a few microseconds that a further increase of the repetition rate has only a negligible influence on the steady-state triplet population; see also Table S1 in the Supporting Information.

The calculated steady-state fraction of RC-LH1 complexes with a closed RC, i.e., an $\mathrm{RC}$ with a $\mathrm{P}^{+}$state, is shown in Figure $9 \mathrm{~A}$ as a function of the fluence for the four repetition rates. For the three smaller repetition rates the fraction of closed RCs is about $6 \%$ at low fluence and rises toward 97\% at higher fluences (in our model this corresponds to all complexes that carry an RC). Yet, the onset of this rise occurs the earlier the higher the repetition rate, and in particular at $81 \mathrm{MHz}$ the fraction of closed RCs is already about $40 \%$ at the lowest fluence applied before it converges toward $97 \%$ at increasing fluences. Plotting the same data as a function of the repetition rate for the $\mathrm{cw}$ equivalent excitation intensities, Figure 9B, allows us to identify three ranges. (i) For $\mathrm{cw}$ equivalent excitation intensities of $8.1 \times 10^{17}$ photons $/\left(\mathrm{s} \cdot \mathrm{cm}^{2}\right)$ and below, the fraction of RC-LH1 complexes with a special pair in the $\mathrm{P}^{+}$ state does not depend on the repetition rate. (ii) At $\mathrm{cw}$ equivalent excitation intensities between $2.6 \times 10^{18}$ and $8.1 \times$ $10^{18}$ photons $/\left(\mathrm{s} \cdot \mathrm{cm}^{2}\right)$ we find a slight decrease of the $\mathrm{P}^{+}$ population for an increase of the repetition from $81 \mathrm{kHz}$ to $810 \mathrm{kHz}$ leveling off for a further increase of the repetition rate. (iii) At the highest shown $\mathrm{cw}$ equivalent excitation intensity of $2.6 \times 10^{19}$ photons $/\left(\mathrm{s} \cdot \mathrm{cm}^{2}\right)$, all RC-LH1 complexes have their special pair in the $\mathrm{P}^{+}$state irrespective of the repetition rate.
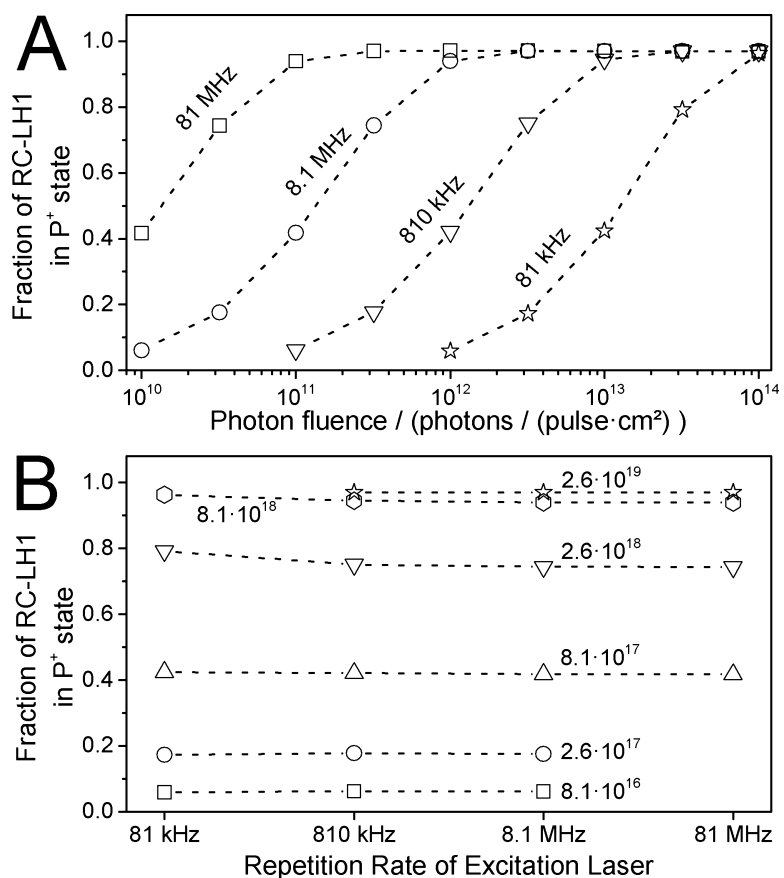

Figure 9. Simulated fraction of RC-LH1 complexes with an oxidized special pair $\left(\mathrm{P}^{+}\right)(\mathrm{A})$ as a function of the fluence and $(\mathrm{B})$ as a function of the repetition rate. The lines serve as a guide for the eye and connect data points of equal repetition rate (A) or equal $\mathrm{cw}$ equivalent excitation intensity (B), given in photons $/\left(\mathrm{s} \cdot \mathrm{cm}^{2}\right)$. Conversions into other dimensions are provided in the Supporting Information, Table S2.

The relative decrease of the $\mathrm{P}^{+}$population between $81 \mathrm{kHz}$ and $810 \mathrm{kHz}$ at elevated $\mathrm{cw}$ equivalent excitation intensities can be rationalized as follows. Only at repetition rates higher than $810 \mathrm{kHz}$ do we find a significant steady-state triplet population. If for these rates the excitation intensity is sufficiently large to have a singlet and a triplet state on the same LH1 ring, STA occurs. As a consequence the number of excitations that can be transferred to the $\mathrm{RC}$ is reduced, which in turn also reduces the population of closed RCs. The threshold for the onset of this effect can be deduced from Figure 9B. For excitation intensities up to $8.1 \times 10^{17}$ photons $/\left(\mathrm{s} \cdot \mathrm{cm}^{2}\right)$ the relative triplet population is about $10^{-4}$ (see Figure $8 \mathrm{~A}$ and Table S1, Supporting Information), which is too small to make a significant impact via STA on the $\mathrm{P}^{+}$population. On the other hand, for $\mathrm{cw}$ excitation intensities above $8.1 \times 10^{18}$ photons $/\left(\mathrm{s} \cdot \mathrm{cm}^{2}\right)$, STA cannot outcompete the rapid buildup of the $\mathrm{P}^{+}$population, so that the latter one saturates. Hence the influence of STA on the $\mathrm{P}^{+}$population is only significant for $\mathrm{cw}$ excitation intensities between $8.1 \times 10^{17}$ photons $/\left(\mathrm{s} \cdot \mathrm{cm}^{2}\right)$ and $8.1 \times 10^{18}$ photons/ $\left(\mathrm{s} \cdot \mathrm{cm}^{2}\right)$. Interestingly, this corresponds to a relative triplet population in the order of $10^{-3}-10^{-4}$ per LH1 ring (see Figure $8 \mathrm{~A}$ and Table S1, Supporting Information), which already makes a detectable impact on the fluorescence decay of isolated RC-LH1 complexes.

\section{CONCLUSION}

We have described a study that deals with the fluorescence decay of isolated RC-LH1 complexes from Rhodopseudomonas palustris on a picosecond time scale. The fluorescence transients were recorded as a function of the photon fluence and the repetition rate of the excitation laser, both of which have been varied over several orders of magnitude. This allowed us to 
identify the simultaneous presence of RC-LH1 complexes in our sample that have an open RC (special pair in the neutral state $\mathrm{P}$ ), those that have a closed RC (special pair in the oxidized state $\mathrm{P}^{+}$), and those that completely lack a reaction center, featuring characteristic fluorescence lifetimes of 40, 200, and 600 ps, respectively. These findings were corroborated by control experiments with reducing or oxidizing agents. For a quantitative analysis of the data we performed elaborate simulations using a global master equation approach based on a microstate description of RC-LH1 and achieved very good agreement between the simulated and the measured transients. This model allows us to predict the relative population triplet states on LH1 ( $\left.{ }^{3} \mathrm{Car}^{*}\right)$ as well as the fraction of closed RCs $\left(\mathrm{P}^{+}\right)$as functions of the photon fluence and the repetition rate of the excitation.

\section{ASSOCIATED CONTENT}

\section{S Supporting Information}

Relation between repetition rate and consecutive pulses exciting the sample, data on the convergence of the simulations, the influence of $Q_{B}$ being absent, the variation of the fraction of RC-less LH1, the variation of the fraction of fresh RC-LH1, numerical input for Figures $7 \mathrm{~b}$ and $8 \mathrm{~b}$, and a conversion table for fluences at different repetition rates into different measures of $\mathrm{cw}$ excitation intensity. This material is available free of charge via the Internet at http://pubs.acs.org.

\section{AUTHOR INFORMATION}

\section{Corresponding Authors}

*(J.K.) E-mail: juergen.koehler@uni-bayreuth.de.

*(G.M.U.) E-mail: matthias.ullmann@uni-bayreuth.de.

\section{Author Contributions}

"(S.R.B. and L.M.) These authors contributed equally to this work.

Notes

The authors declare no competing financial interest.

\section{ACKNOWLEDGMENTS}

Financial support by the Deutsche Forschungsgemeinschaft (DFG) within projects GRK 1640 (S.R.B., L.M., G.M.U., and J.K.), and KO 1359/21-1 (S.R.B. and J.K.) is thankfully acknowledged. R.J.C. thanks BBSRC for support.

\section{REFERENCES}

(1) McDermott, G.; Prince, S. M.; Freer, A. A.; HawthornthwaiteLawless, A. M.; Papiz, M. Z.; Cogdell, R. J.; Isaacs, N. W. Crystal Structure of an Integral Membrane Light-Harvesting Complex from Photosynthetic Bacteria. Nature 1995, 374, 517-521.

(2) McLuskey, K.; Prince, S. M.; Cogdell, R. J.; Isaacs, N. W. The Crystallographic Structure of the B800-820 LH3 Light-Harvesting Complex from the Purple Bacteria Rhodopseudomonas Acidophila Strain 7050. Biochemistry 2001, 40, 8783-8789.

(3) Koepke, J.; Hu, X.; Muenke, C.; Schulten, K.; Michel, H. The Crystal Structure of the Light-Harvesting Complex II (B800-850) from Rhodospirillum Molischianum. Structure 1996, 4, 581-597.

(4) Roszak, A. W. Crystal Structure of the RC-LH1 Core Complex from Rhodopseudomonas Palustris. Science 2003, 302, 1969-1972.

(5) Qian, P.; Neil Hunter, C.; Bullough, P. A. The 8.5 Å Projection Structure of the Core RC-LH1-PufX Dimer of Rhodobacter Sphaeroides. J. Mol. Biol. 2005, 349, 948-960.

(6) Niwa, S.; Yu, L.-J.; Takeda, K.; Hirano, Y.; Kawakami, T.; WangOtomo, Z.-Y.; Miki, K. Structure of the LH1-RC Complex from Thermochromatium Tepidum at 3.0 Å. Nature 2014, 508, 228-232.
(7) The Purple Phototrophic Bacteria; Hunter, C. N., Fevzi, D., Thurnauer, M. C., Beatty, J. T., Eds.; Advances in Photosynthesis and Respiration; Springer: 2008; Vol. 28.

(8) Deisenhofer, J.; Epp, O.; Miki, K.; Huber, R.; Michel, H. Structure of the Protein Subunits in the Photosynthetic Reaction Centre of Rhodopseudomonas Viridis at $3 \AA \AA$ Resolution. Nature 1985, 318, 618-624.

(9) Allen, J. P.; Feher, G.; Yeates, T. O.; Komiya, H.; Rees, D. C. Structure of the Reaction Center from Rhodobacter Sphaeroides R-26: The Protein Subunits. Proc. Natl. Acad. Sci. U. S. A. 1987, 84, 61626166.

(10) Koepke, J.; Krammer, E.-M.; Klingen, A. R.; Sebban, P.; Ullmann, G. M.; Fritzsch, G. pH Modulates the Quinone Position in the Photosynthetic Reaction Center from Rhodobacter Sphaeroides in the Neutral and Charge Separated States. J. Mol. Biol. 2007, 371, 396409 .

(11) Feher, G.; Allen, J. P.; Okamura, M. Y.; Rees, D. C. Structure and Function of Bacterial Photosynthetic Reaction Centres. Nature 1989, 339, 111-116.

(12) Scheuring, S.; Busselez, J.; Levy, D. Structure of the Dimeric PufX-Containing Core Complex of Rhodobacter Blasticus by in Situ Atomic Force Microscopy. J. Biol. Chem. 2005, 280, 1426-1431.

(13) Qian, P.; Bullough, P. A.; Hunter, C. N. Three-Dimensional Reconstruction of a Membrane-Bending Complex: The RC-LH1-PufX Core Dimer of Rhodobacter Sphaeroides. J. Biol. Chem. 2008, 283, 14002-14011.

(14) Richter, M. F.; Baier, J.; Prem, T.; Oellerich, S.; Francia, F.; Venturoli, G.; Oesterhelt, D.; Southall, J.; Cogdell, R. J.; Köhler, J. Symmetry Matters for the Electronic Structure of Core Complexes from Rhodopseudomonas Palustris and Rhodobacter Sphaeroides PufX'. Proc. Natl. Acad. Sci. U. S. A. 2007, 104, 6661-6665.

(15) Richter, M. F.; Baier, J.; Southall, J.; Cogdell, R. J.; Oellerich, S.; Köhler, J. Refinement of the X-Ray Structure of the RC-LH1 Core Complex from Rhodopseudomonas Palustris by Single-Molecule Spectroscopy. Proc. Natl. Acad. Sci. U. S. A. 2007, 104, 20280-20284.

(16) Ermler, U.; Fritzsch, G.; Buchanan, S. K.; Michel, H. Structure of the Photosynthetic Reaction Centre from Rhodobacter Sphaeroides at $2.65 \AA$ Resolution: Cofactors and Protein-Cofactor Interactions. Structure 1994, 2, 925-936.

(17) Olson, T. L.; Williams, J. C.; Allen, J. P. The Three-Dimensional Structures of Bacterial Reaction Centers. Photosynth. Res. 2014, 120, 87-98.

(18) Möbius, K.; Lubitz, W.; Savitsky, A. Photo-Induced Electron Spin Polarization in Chemical and Biological Reactions: Probing Structure and Dynamics of Transient Intermediates by Multifrequency EPR Spectroscopy. Appl. Magn. Reson. 2011, 41, 113-143.

(19) van der Est, A.; Reiss-Husson, F.; Stehlik, D. An Investigation of the Light-Induced Spin Polarization in Reaction Centres of Rhodobacter Sphaeroides Y. Photosynth. Res. 1998, 55, 217-225.

(20) Prisner, T. F.; van der Est, A.; Bittl, R.; Lubitz, W.; Stehlik, D.; Möbius, K. Time-Resolved W-Band (95 GHz) EPR Spectroscopy of Zn-Substituted Reaction Centers of Rhodobacter Sphaeroides R-26. Chem. Phys. 1995, 194, 361-370.

(21) Marchanka, A.; Paddock, M.; Lubitz, W.; van Gastel, M. LowTemperature Pulsed EPR Study at $34 \mathrm{GHz}$ of the Triplet States of the Primary Electron Donor $\mathrm{P}_{865}$ and the Carotenoid in Native and Mutant Bacterial Reaction Centers of Rhodobacter Sphaeroides. Biochemistry 2007, 46, 14782-14794.

(22) Bittl, R.; Zech, S. G. Pulsed EPR Study of Spin-Coupled Radical Pairs in Photosynthetic Reaction Centers: Measurement of the Distance Between $\mathrm{P}^{\bullet+}{ }_{700}$ and $\mathrm{A}^{\bullet-}{ }_{1}$ in Photosystem I and between $\mathrm{P}^{\bullet+}{ }_{865}$ and $\mathrm{Q}^{\bullet-}$ A in Bacterial Reaction Centers. J. Phys. Chem. B 1997, $101,1429-1436$.

(23) Bittl, R; Weber, S. Transient Radical Pairs Studied by TimeResolved EPR. Biochim. Biophys. Acta, Bioenerg. 2005, 1707, 117-126.

(24) Fajer, J.; Brune, D. C.; Davis, M. S.; Forman, A.; Spaulding, L. D. Primary Charge Separation in Bacterial Photosynthesis: Oxidized Chlorophylls and Reduced Pheophytin. Proc. Natl. Acad. Sci. U. S. A. 1975, 72, 4956-4960. 
(25) Finkele, U.; Lauterwasser, C.; Zinth, W.; Gray, K. A.; Oesterhelt, D. Role of Tyrosine M210 in the Initial Charge Separation of Reaction Centers of Rhodobacter Sphaeroides. Biochemistry 1990, 29, 85178521.

(26) Stilz, H. U.; Finkele, U.; Holzapfel, W.; Lauterwasser, C.; Zinth, W.; Oesterhelt, D. Influence of M Subunit Thr222 and Trp252 on Quinone Binding and Electron Transfer in Rhodobacter Sphaeroides Reaction Centres. Eur. J. Biochem. 1994, 223, 233-242.

(27) Dominguez, P. N.; Himmelstoss, M.; Michelmann, J.; Lehner, F. T.; Gardiner, A. T.; Cogdell, R. J.; Zinth, W. Primary Reactions in Photosynthetic Reaction Centers of Rhodobacter Sphaeroides - Time Constants of the Initial Electron Transfer. Chem. Phys. Lett. 2014, 601, 103-109.

(28) Beekman, L. M. P.; Visschers, R. W.; Monshouwer, R.; HeerDawson, M.; Mattioli, T. A.; McGlynn, P.; Hunter, C. N.; Robert, B.; van Stokkum, I. H. M. Time-Resolved and Steady-State Spectroscopic Analysis of Membrane-Bound Reaction Centers from Rhodobacter Sphaeroides: Comparisons with Detergent-Solubilized Complexes. Biochemistry 1995, 34, 14712-14721.

(29) Fleming, G. R.; Martin, J. L.; Breton, J. Rates of Primary Electron Transfer in Photosynthetic Reaction Centres and Their Mechanistic Implications. Nature 1988, 333, 190-192.

(30) Williams, J. C.; Alden, R. G.; Murchison, H. A.; Peloquin, J. M.; Woodbury, N. W.; Allen, J. P. Effects of Mutations near the Bacteriochlorophylls in Reaction Centers from Rhodobacter Sphaeroides. Biochemistry 1992, 31, 11029-11037.

(31) Meyer, M.; Scheer, H. Reaction Centers of Rhodobacter Sphaeroides R26 Containing C-3 Acetyl and Vinyl (bacterio)pheophytins at Sites $\mathrm{H}_{\mathrm{A}, \mathrm{B}}$. Photosynth. Res. 1995, 44, 55-65.

(32) Zinth, W.; Wachtveitl, J. The First Picoseconds in Bacterial Photosynthesis - Ultrafast Electron Transfer for the Efficient Conversion of Light Energy. ChemPhysChem 2005, 6, 871-880.

(33) Blankenship, R. E. Molecular Mechanisms of Photosynthesis; Blackwell Science: Oxford; Malden, MA, 2002.

(34) Vredenberg, W. J.; Duysens, L. N. M. Transfer of Energy from Bacteriochlorophyll to a Reaction Centre During Bacterial Photosynthesis. Nature 1963, 197, 355-357.

(35) Clayton, R. K. Fluorescence from Major and Minor Bacteriochlorophyll Components in Vivo. Photochem. Photobiol. 1966, 5, 679-688.

(36) Heathcote, P.; Clayton, R. K. Reconstituted Energy Transfer from Antenna Pigment-Protein to Reaction Centres Isolated from Rhodopseudomonas Sphaeroides. Biochim. Biophys. Acta, Bioenerg. 1977, 459, 506-515.

(37) Borisov, A. Y.; Godik, V. I. Energy Transfer in Bacterial Photosynthesis. J. Bioenerg. 1972, 3, 211-220.

(38) Godik, V. I.; Borisov, A. Y. Excitation Trapping by Different States of Photosynthetic Reaction Centres. FEBS Lett. 1977, 82, 355358.

(39) Ritz, T.; Park, S.; Schulten, K. Kinetics of Excitation Migration and Trapping in the Photosynthetic Unit of Purple Bacteria. J. Phys. Chem. B 2001, 105, 8259-8267.

(40) Strümpfer, J.; Schulten, K. Excited State Dynamics in Photosynthetic Reaction Center and Light Harvesting Complex 1. J. Chem. Phys. 2012, 137, 065101.

(41) Visscher, K. J.; Bergström, H.; Sundström, V.; Hunter, C. N.; Grondelle, R. V. Temperature Dependence of Energy Transfer from the Long Wavelength Antenna BChl-896 to the Reaction Center in Rhodospirillum Rubrum, Rhodobacter Sphaeroides (w.t. and M21 Mutant) from 77 to $177 \mathrm{~K}$, Studied by Picosecond Absorption Spectroscopy. Photosynth. Res. 1989, 22, 211-217.

(42) Bergström, H.; van Grondelle, R.; Sundström, V. Characterization of Excitation Energy Trapping in Photosynthetic Purple Bacteria at 77 K. FEBS Lett. 1989, 250, 503-508.

(43) Valkunas, L.; Liuolia, V.; Freiberg, A. Picosecond Processes in Chromatophores at Various Excitation Intensities. Photosynth. Res. 1991, 27, 83-95.
(44) Van Grondelle, R.; Dekker, J. P.; Gillbro, T.; Sundstrom, V. Energy Transfer and Trapping in Photosynthesis. Biochim. Biophys. Acta, Bioenerg. 1994, 1187, 1-65.

(45) Sundström, V.; Pullerits, T.; van Grondelle, R. Photosynthetic Light-Harvesting: Reconciling Dynamics and Structure of Purple Bacterial LH2 Reveals Function of Photosynthetic Unit. J. Phys. Chem. B 1999, 103, 2327-2346.

(46) Timpmann, K.; Zhang, F. G.; Freiberg, A.; Sundström, V. Detrapping of Excitation Energy from the Reaction Centre in the Photosynthetic Purple Bacterium Rhodospirillum Rubrum. Biochim. Biophys. Acta, Bioenerg. 1993, 1183, 185-193.

(47) Timpmann, K.; Freiberg, A.; Sundström, V. Energy Trapping and Detrapping in the Photosynthetic Bacterium Rhodopseudomonas Viridis: Transfer-to-Trap-Limited Dynamics. Chem. Phys. 1995, 194, $275-283$.

(48) Xiao, W.; Lin, S.; Taguchi, A. K. W.; Woodbury, N. W. Femtosecond Pump-Probe Analysis of Energy and Electron Transfer in Photosynthetic Membranes of Rhodobacter Capsulatus. Biochemistry 1994, 33, 8313-8322.

(49) Bernhardt, K.; Trissl, H.-W. Escape Probability and Trapping Mechanism in Purple Bacteria: Revisited. Biochim. Biophys. Acta, Bioenerg. 2000, 1457, 1-17.

(50) Abdourakhmanov, I. A.; Danielius, R. V.; Razjivin, A. P. Efficiency of Excitation Trapping by Reaction Centres of Complex B890 from Chromatium Minutissimum. FEBS Lett. 1989, 245, 47-50.

(51) Freiberg, A.; Godik, V. I.; Pullerits, T.; Timpman, K. Picosecond Dynamics of Directed Excitation Transfer in Spectrally Heterogeneous Light-Harvesting Antenna of Purple Bacteria. Biochim. Biophys. Acta, Bioenerg. 1989, 973, 93-104.

(52) Sebban, P.; Moya, I. Fluorescence Lifetime Spectra of in Vivo Bacteriochlorophyll at Room Temperature. Biochim. Biophys. Acta, Bioenerg. 1983, 722, 436-442.

(53) Sundström, V.; van Grondelle, R.; Bergström, H.; Åkesson, E.; Gillbro, T. Excitation-Energy Transport in the Bacteriochlorophyll Antenna Systems of Rhodospirillum Rubrum and Rhodobacter Sphaeroides, Studied by Low-Intensity Picosecond Absorption Spectroscopy. Biochim. Biophys. Acta, Bioenerg. 1986, 851, 431-446.

(54) Freiberg, A. Coupling of Antennas to Reaction Centers. In Anoxigenic Photosynthetic Bacteria; Blankenship, R. E., Madigan, M. T., Bauer, C. E., Eds.; Kluwer Academic Publishers: 1995; pp 385-398.

(55) Van Grondelle, R.; Bergström, H.; Sundström, V.; Gillbro, T. Energy Transfer within the Bacteriochlorophyll Antenna of Purple Bacteria at $77 \mathrm{~K}$, Studied by Picosecond Absorption Recovery. Biochim. Biophys. Acta, Bioenerg. 1987, 894, 313-326.

(56) Freiberg, A.; Allen, J. P.; Williams, J. C.; Woodbury, N. W. Energy Trapping and Detrapping by Wild Type and Mutant Reaction Centers of Purple Non-Sulfur Bacteria. Photosynth. Res. 1996, 48, 309319.

(57) Sebban, P.; Jolchine, G.; Moya, I. Spectra of Fluorescence Lifetime and Intensity of Rhodopseudomonas Sphaeroides at Room and Low Temperature. Comparison Between the Wild Type, the C 71 Reaction Center-Less Mutant and the B800-850 Pigment-Protein Complex. Photochem. Photobiol. 1984, 39, 247-253.

(58) Freiberg, A.; Timpmann, K. Picosecond Fluorescence Spectroscopy of Light-Harvesting Antenna Complexes from Rhodospirillum Rubrum in the 300-4 K Temperature Range. Comparison with the Data on Chromatophores. J. Photochem. Photobiol., B 1992, 15, 151158.

(59) Timpmann, K.; Freiberg, A.; Godik, V. I. Picosecond Kinetics of Light Excitations in Photosynthetic Purple Bacteria in the Temperature Range of 300-4 K. Chem. Phys. Lett. 1991, 182, 617-622.

(60) Borisov, A. Y.; Freiberg, A.; Godik, V.; Rebane, K.; Timpmann, K. Kinetics of Picosecond Bacteriochlorophyll Luminescence in Vivo as a Function of the Reaction Center State. Biochim. Biophys. Acta, Bioenerg. 1985, 807, 221-229.

(61) Stahl, A. D.; Crouch, L. I.; Jones, M. R.; van Stokkum, I.; van Grondelle, R.; Groot, M. L. Role of PufX in Photochemical Charge Separation in the RC-LH1 Complex from Rhodobacter Sphaeroides: An 
Ultrafast Mid-IR Pump-Probe Investigation. J. Phys. Chem. B 2012, 116, 434-444.

(62) Pflock, T. J.; Oellerich, S.; Southall, J.; Cogdell, R. J.; Ullmann, G. M.; Köhler, J. The Electronically Excited States of LH2 Complexes from Rhodopseudomonas Acidophila Strain 10050 Studied by TimeResolved Spectroscopy and Dynamic Monte Carlo Simulations. I. Isolated, Non-Interacting LH2 Complexes. J. Phys. Chem. B 2011, 115, 8813-8820.

(63) Pflock, T. J.; Oellerich, S.; Krapf, L.; Southall, J.; Cogdell, R. J.; Ullmann, G. M.; Köhler, J. The Electronically Excited States of LH2 Complexes from Rhodopseudomonas Acidophila Strain 10050 Studied by Time-Resolved Spectroscopy and Dynamic Monte Carlo Simulations. II. Homo-Arrays Of LH2 Complexes Reconstituted Into Phospholipid Model Membranes. J. Phys. Chem. B 2011, 115, 8821-8831.

(64) Clayton, R. K. Spectroscopic Analysis of Bacteriochlorophylls in Vitro and in Vivo*. Photochem. Photobiol. 1966, 5, 669-677.

(65) Becker, T.; Ullmann, R. T.; Ullmann, G. M. Simulation of the Electron Transfer between the Tetraheme Subunit and the Special Pair of the Photosynthetic Reaction Center Using a Microstate Description. J. Phys. Chem. B 2007, 111, 2957-2968.

(66) Bombarda, E.; Ullmann, G. M. Continuum Electrostatic Investigations of Charge Transfer Processes in Biological Molecules Using a Microstate Description. Faraday Discuss. 2011, 148, 173.

(67) Müller, M. G.; Drews, G.; Holzwarth, A. R. Excitation Transfer and Charge Separation Kinetics in Purple Bacteria. (1) Picosecond Fluorescence of Chromatophores from Rhodobacter Capsulatus Wild Type. Biochim. Biophys. Acta, Bioenerg 1993, 1142, 49-58.

(68) Law, C. J.; Cogdell, R. J. The Effect of Chemical Oxidation on the Fluorescence of the LH1 (B880) Complex from the Purple Bacterium Rhodobium Marinum. FEBS Lett. 1998, 432, 27-30.

(69) Valkunas, L.; Akesson, E.; Pullerits, T.; Sundström, V. Energy Migration in the Light-Harvesting Antenna of the Photosynthetic Bacterium Rhodospirillum Rubrum Studied by Time-Resolved Excitation Annihilation at $77 \mathrm{~K}$. Biophys. J. 1996, 70, 2373-2379.

(70) Trinkunas, G.; Herek, J.; Polívka, T.; Sundström, V.; Pullerits, T. Exciton Delocalization Probed by Excitation Annihilation in the LightHarvesting Antenna LH2. Phys. Rev. Lett. 2001, 86, 4167-4170.

(71) Monger, T. G.; Cogdell, R. J.; Parson, W. W. Triplet States of Bacteriochlorophyll and Carotenoids in Chromatophores of Photosynthetic Bacteria. Biochim. Biophys. Acta, Bioenerg. 1976, 449, 136153.

(72) Juan, F.; Xue-feng, L.; Yuan, L. Selective Distribution and Physiological Function of Heterogeneous Carotenoids in Purple Photosynthetic Bacterium Rhodopseudomonas palustris. Spectrosc. Spectr. Anal. 2008, 28, 1459-1463.

(73) Kakitani, Y.; Akahane, J.; Ishii, H.; Sogabe, H.; Nagae, H.; Koyama, Y. Conjugation-Length Dependence of the $T_{1}$ Lifetimes of Carotenoids Free in Solution and Incorporated into the LH2, LH1, RC, and RC-LH1 Complexes: Possible Mechanisms of Triplet-Energy Dissipation. Biochemistry 2007, 46, 2181-2197.

(74) Bittl, R.; Schlodder, E.; Geisenheimer, I.; Lubitz, W.; Cogdell, R. J. Transient EPR and Absorption Studies of Carotenoid Triplet Formation in Purple Bacterial Antenna Complexes. J. Phys. Chem. B 2001, 105, 5525-5535.

(75) Graige, M. S.; Feher, G.; Okamura, M. Y. Conformational Gating of the Electron Transfer Reaction $\mathrm{Q}_{A}{ }^{-\bullet} \mathrm{Q}_{B} \rightarrow \mathrm{Q}_{A} \mathrm{Q}_{B}{ }^{-\bullet}$ in Bacterial Reaction Centers of Rhodobacter Sphaeroides Determined by a Driving Force Assay. Proc. Natl. Acad. Sci. U. S. A. 1998, 95, 11679-11684.

(76) Shinkarev, V. P.; Wraight, C. A. Electron and Proton Transfer in the Acceptor Quinone Complex of Reaction Centers of Phototrophic Bacteria. In The Photosynthetic Reaction Center; Deisenhofer, J., Norris, J. R., Eds.; Academic Press Inc.: 1993; Vol. I, pp 193-256.

(77) Chuang, J. I.; Boxer, S. G.; Holten, D.; Kirmaier, C. High Yield of M-Side Electron Transfer in Mutants of Rhodobacter Capsulatus Reaction Centers Lacking the L-Side Bacteriopheophytin. Biochemistry 2006, 45, 3845-3851.
(78) Carter, B.; Boxer, S. G.; Holten, D.; Kirmaier, C. Trapping the P $+\mathrm{BL}-$ Initial Intermediate State of Charge Separation in Photosynthetic Reaction Centers from Rhodobacter Capsulatus. Biochemistry 2009, 48, 2571-2573.

(79) Pan, J.; Lin, S.; Woodbury, N. W. Bacteriochlorophyll ExcitedState Quenching Pathways in Bacterial Reaction Centers with the Primary Donor Oxidized. J. Phys. Chem. B 2012, 116, 2014-2022. 